\title{
Introducción al Dossier Marcadores óseos de ¿actividad?: Aproximaciones y limitaciones desde estudios \\ bioarqueológicos
}

\author{
Skeletal markers of occupational stress? Approaches and constraints \\ from bioarchaeological studies
}

RMA

Dossier

Bárbara Mazza* y Mariana Fabra**

*Instituto Nacional de Antropología y Pensamiento Latinoamericano (INAPL), CONICET. E-mail: barbara_mazza@yahoo.com.ar; ** Instituto de Antropología de Córdoba (IDACOR)CONICET, Museo de Antropología (Facultad de Filosofía y Humanidades, Universidad Nacional de Córdoba).E-mail: marianafabra@gmail.com

Este dossier reúne los trabajos presentados en el simposio que tuvo el mismo nombre, y fue coordinado por nosotras, en el marco del XIV Congreso de la Asociación Latinoamericana de Antropología Biológica (ALAB) durante los días 18 al 21 de Octubre de 2016 en la ciudad de Tacuarembó, Uruguay. En él se presentaron seis trabajos donde se discutieron y aplicaron diversos métodos tanto para el relevamiento como para el análisis estadístico de marcadores óseos que pueden brindar información acerca de niveles de actividad física junto a sus implicancias sociales, culturales y adaptativas. Se presentaron los resultados obtenidos y se discutieron sus alcances y limitaciones, tomando como casos de estudio poblaciones arqueológicas de Chile y Argentina y contemporáneas de Portugal.

Los "marcadores de estrés músculo-esqueletal", o bien los "marcadores óseos de actividad", abarcan una serie de rasgos óseos utilizados frecuentemente para inferir el estilo de vida de poblaciones humanas pasadas. Entre ellos,los cambios entésicos (modificaciones óseas que ocurren en las zonas de inserción de músculos, tendones o ligamentos), así como la osteoartrosis o enfermedad degenerativa articular (cambios degenerativos que acontecen en las articulaciones) son los indicadores más utilizados por varios investigadores a nivel mundial. Tradicionalmente estos cambios morfológicos fueron considerados como el producto de una fuerza o presión sobre un área ósea que excedía al límite elástico del hueso, provocando así modificaciones en la morfología del tejido óseo y estimulando la formación de hueso nuevo.

En lo que respecta a la Arqueología, desde fines de 1980 y 1990 ha habido un aumento en el interés por el estudio de la variabilidad en la morfología de las entesis y de las articulaciones, tanto a escala individual como poblacional, para reconstruir los comportamientos de sociedades prehistóricas e históricas. A los fines de poder estudiar los cambios entésicos, osteoartrosis y otras patologías relacionadas, se desarrollaron varias metodologías macroscópicas cualitativas y cuantitativas, bajo el supuesto que las diferentes expresiones morfológicas son un correlato directo de la acumulación de estrés repetitivo en las entesis y en las articulaciones. Sin embargo, a partir de estudios médicos se ha subrayado la importancia de tener en cuenta otros factores intrínsecos al individuo que activan el remodelamiento óseo y que, por ende, son independientes de estresores mecánicos, como ser la genética, la edad, el tamaño corporal y muscular de los individuos, las hormonas, la presencia de traumas y de condiciones patológicas, entre otros.

El trabajo de Viviana Llagostera Leyton analiza la organización social de una población jerárquica agrícola y pastoril de San Pedro de Atacama (Chile) a través de la presencia de la enfermedad degenerativa articular. A raíz de la ausencia

Recibido 12-10-2016. Recibido con correcciones 17-08-2017. Aceptado 10-09-2017 
de diferencias en la manifestación de dicha patología entre los entierros de estatus alto y bajo, llega a la conclusión de que el estatus social no estaría relacionado con el desarrollo de la enfermedad degenerativa articular.

El artículo de Soledad Salega y Mariana Fabra evalúa la relación entre los cambios entésicos de miembros inferiores y dos tipos de terreno, llano o montañoso, bajo la hipótesis de que los individuos de terrenos montañosos presentan menores cambios entésicos que aquellos de terrenos llanos debido a una mejor adaptación biomecánica en los primeros, siguiendo la propuesta de Acosta et al. (2017). Para esto analizan una muestra de individuos de las regiones de Sierra y Llanuras de la provincia de Córdoba (Argentina). Sus resultados corroboran su hipótesis, ya que los individuos de las llanuras presentan mayores cambios entésicos que los de terrenos montañosos. Sin embargo, las autoras advierten que si bien sus hallazgos pueden explicarse desde un punto de vista adaptativo a la carga biomecánica, también puede deberse a una repetición de actividades físicas exigentes.

El artículo de Soledad Salega, Charlotte Henderson y Ana María Silva evalúa el aumento en la urbanización y en la especialización laboral entre el período medieval y moderno en Portugal, a través del análisis de los cambios entésicos. Sus resultados señalan que la actividad es un factor que influyó en la morfología de las entesis, pero que, sin embargo, también deben tenerse en cuenta el sexo y la edad de los individuos como otros factores explicativos.

El trabajo de Bárbara Mazza evalúa una serie de pruebas estadísticas bivariadas y multivariadas para el estudio de diferencias sexuales en los cambios entésicos de una muestra de poblaciones cazadoras-recolectoras que habitaron el sur de la provincia de Entre Ríos y el norte de Buenos Aires (Argentina). Si bien todos los análisis mostraron la presencia de diferencias significativas entre ambos sexos, recomienda el empleo de pruebas estadísticas donde se pueda controlar los efectos de varias variables al mismo tiempo.

Por otra parte, el trabajo de Eva Peralta aborda el estudio de las estrategias de subsistencia y movilidad de poblaciones humanas que habitaron el sur de Mendoza (Argentina), a partir del analisis de cambios entésicos y degenerativos y valores isotópicos de oxígeno $\left(\delta^{18} \mathrm{O}\right)$. La hipótesis propuesta era que la reducción de la movilidad residencial, derivada del cambio hacia una estrategia productiva de alimentos alrededor de los 2000 años AP, habría derivado en a) un menor desarrollo de los cambios entésicos y lesiones asociadas a osteoartrosis en miembros inferiores y b) poca variabilidad interindividual para los valores de $\delta^{18} \mathrm{O}$. Los resultados no fueron los esperados, proponiendo entonces que si bien estos marcadores óseos no permitirían discriminar entre distintos tipos de estrategias de subsistencia, si permitirían avanzar en torno a hipótesis sobre la movilidad de las poblaciones.

Finalmente, el trabajo de Josefina Urrea Navarrete, Pedro Andrade, Sonia Parra, Oscar Achiardi, Hernán Salinas y Mary Anne Argo plantea un estudio experimental cinemático como vía para reducir algunos errores en la interpretación de las actividades realizadas por poblaciones arqueológicas. Si bien reconocen limitantes metodológicas y analíticas en este estudio, pudieron ejemplificar el modo en que la postura corporal afecta la activación muscular y variaciones en el porcentaje de esta última para un mismo gesto técnico. También, plantean la necesidad deconocer de forma completa las cadenas operativas para identificar los requerimientos biomecánicos de distintas actividades productivas. Además, debido al origen multivariado de la activación muscular, sugieren que los marcadores óseos de actividad deben ser analizados contextualmente, considerando dimensiones biológicas, ambientales y sociales.

Respecto a las metodologías aplicadas en los distintos trabajos, para el registro de lesiones vinculadas con la enfermedad degenerativa articular, Llagostera Leyton y Peralta han seguido las propuestas basadas en los trabajos de Bass (1971) y Ubelaker (1978), y más recientemente, Luna y colaboradores (2017). Por otra parte, Urrea Navarrete y colaboradores, 
siguieron el protocolo propuesto por SENIAM (Surface Electro Myo Graphyforthe Non-Invasive Assessment of Muscles) para la medición de la activación eléctrica de los músculos por medio de electromiografías superficiales. En cuanto al registro de los cambios entésicos, es interesante mencionar que si bien los distintos autores acuerdan en torno a los conceptos generales para su definición y etiología multicausal (Resnick y Niwayama 1983), no hay un consenso respecto a las metodologías para su registro ni para su posterior análisis estadístico. En los trabajos que forman parte de este Dossier, se han aplicado métodos que relevan la robusticidad de las entesis (Mariotti et al. 2007), así como la robusticidad, lesiones por estrés y exostosis (Hawkey y Merbs 1995) o el nuevo método Coimbra (Henderson et al. 2013, 2016) desarrollado para entesis fibrocartilaginosas. Destacamos en este marco el trabajo de Mazza, donde da cuenta de la necesidad de aplicar análisis estadísticos que permitan controlar el efecto de una serie de variables explicativas sobre la variable dependiente al mismo tiempo, ventaja ofrecida por los modelos lineales generalizados (Henderson y Nikita 2015; Nikita 2014).

Más allá de los resultados particulares alcanzados en cada investigación incluida en este Dossier, que dan cuenta del valor que tienen estos marcadores en discusiones acerca de los niveles de actividad física, o la movilidad de poblaciones arqueológicas, interesa destacar que los mismos, en particular los cambios entesiales, pueden ser explicados atendiendo a diferentes causas. Entre ellos podemos mencionar la adaptación biomecánica o la repetición de actividades físicas exigentes (Salega y Fabra; Llagostera Leyton, este volumen), pero sin descartar la influencia de factores genéticos,la edad y el sexo (Salega et al.; Mazza, este volumen). Se torna necesario, a futuro, alcanzar consensos en torno a los métodos para el registro de distintas entesis, así como establecer los análisis estadísticos apropiados, que permitan controlar distintas variables al mismo tiempo (Mazza, este volumen). De esta forma, el estudio de estos marcadores podrá avanzar hacia estudios comparativos entre sociedades con diferentes tipos de economía, sistemas sociales y políticos.

\section{Bibliografía}

Acosta, M. A., C. Y. Henderson y E. Cuhna. 2017. The effect of terrain on entheseal changes in the lower limbs. International Journal of Osteoarchaeology. DOI: 10.1002/oa.2597

Bass, M. 1971. Human Osteology: a laboratory and field manual of human skeleton. Editor Michel K. Trimble. Missouri Archaeological Society, Columbia

Hawkey, D., C. Merbs. 1995. Activity induced musculoskeletal stress markers (MSM) and subsistence strategy changes among ancient Hudson Bay Eskimos. International Journal of Osteoarchaeology, 5: 324-338.

Henderson, C., V. Mariotti, D. Pany-Kucera, S. VIllotte, y C. Wilczak. 2013. Recording specific entheseal changes of fibrocartilaginous entheses: initial tests using the Coimbra method. International Journal of Osteoarchaeology, 23: 152-162.

Henderson, C., E. Nikita. 2015. Accounting for multiple effects and the problem of small sample sizes in osteology: A case study focusing on entheseal changes. Archaeological Anthropological Science, DOI 10.1007/s12520-015-0256-1.

Henderson, C., V. Mariotti, D. Pany-Kucera, S. Villotte y C. Wilczak. 2016. The new "Coimbra method": a biologically appropriate method for recording specific features of fibrocartilaginous entheseal changes. International Journal of Osteoarchaeology, 26(5): 925-932. 
Mariotti, V., F. Facchini y M. Giovanna Belcastro. 2007. The study of entheses: proposal of a standardizedscoring method for twenty-three entheses of the postcranial skeleton. CollegiumAntropologicum, 31: 291-313.

Luna, L., C. Aranda y A. Amorim Alves. 2017. Reflexiones sobre el relevamiento y análisis comparativo de patologías osteoarticulares en restos esqueletales humanos. Revista Argentina de AntropologíaBiológica, 19(1): 1-8.

Nikita, E. 2014. The use of generalized linear models and generalized estimating equations in bioarchaeological studies. American Journal of Physical Anthropology, 153: 473-483.

Resnick, D., G. Niwayama. 1983. Enthesis and enthesopathy: anatomical, pathological and radiological correlation. Radiology, $146(1): 1-9$

Ubelaker, D. 1978. Human skeletal remains: excavation, analysis, interpretation. Smithsonian Institution, Taraxacum, Washington, DC. 